\title{
A Solution Method for Longitudinal Vibrations of Functionally Graded Nanorods
}

\author{
Büşra Uzun ${ }^{a^{*}}$, M. Özgür Yayll ${ }^{b}$ \\ ${ }^{a, b}$ Bursa Uludag University, Engineering Faculty, Department of Civil Engineering \\ Division of Mechanics, Bursa-TURKEY \\ ${ }^{*}$ E-mail address: buzun@uludag.edu.tr ${ }^{\mathrm{a}^{*}}, \underline{\text { ozguryayli@ } @ \text { uludag.edu.tr }}{ }^{\mathrm{b}}$
}

ORCID numbers of authors:

0000-0002-7636-7170 ${ }^{a}, 0000-0003-2231-170 \mathrm{X}^{b}$

Received date: 19.08 .2020

Accepted date: 06.10 .2020

\begin{abstract}
In the present study, a nonlocal finite element formulation of free longitudinal vibration is derived for functionally graded nano-sized rods. Size dependency is considered via Eringen's nonlocal elasticity theory. Material properties, Young's modulus and mass density, of the nano-sized rod change in the thickness direction according to the power-law. For the examined FG nanorod finite element, the axial displacement is specified with a linear function. The stiffness and mass matrices of functionally graded nano-sized rod are found by means of interpolation functions. Functionally graded nanorod is considered with clamped-free boundary condition and its longitudinal vibration analysis is performed.
\end{abstract}

Keywords: Nonlocal elasticity theory, Functionally graded materials, Nanorod, Finite element method, Vibration

\section{Introduction}

One of the popular structures of recent times is functionally graded (FG) composite materials. The difference of these materials which are usually a combination of metal and ceramic from traditional laminated composites is that the smooth changing of material properties. In functionally graded materials, the material properties like Young's modulus, density, shear modulus etc. change according to a certain rule continuously along at least one direction. Thanks to this smooth property changing, functionally graded materials have been precious for many applications such as biomedical, chemistry, electronics, optics, aircraft, space vehicles and biology etc. [1,2]. In addition, functionally graded structures have attracted considerable attention in models of nano/micro mechanics. The studies on functionally graded nano/micro structures such as FG nanoplate [3-7], FG nanobeam [8-14], FG nanorod [14-18] have been presented by researchers in recent years. 
In addition to the analytical solution [19,20], many other methods like discrete singular convolution method [21,22], polynomial differential quadrature method [23], finite difference method, finite element method [24] etc. have been used by researchers to solve a problem. In this study, a finite element formulation for free longitudinal vibration behavior of functionally graded nanorod is presented. Small-scale effect of the functionally graded nanorod is discussed based on the nonlocal elasticity theory. The nonlocal elasticity theory has an additional small-scale parameter (nonlocal parameter) and thanks to this nonlocal parameter the small-scale effects occurring in nano/micro-sized structures can be evaluated. The nonlocal elasticity theory has become a frequently performed theory in nanomechanics and micromechanics, as it allows the consideration of small-scale effects. In addition, articles using finite element method to examine the behavior of size-dependent microstructures/nanostructures such as vibration [25-30], buckling [29-32] and bending [2930, 33-35] are also found in the literature.
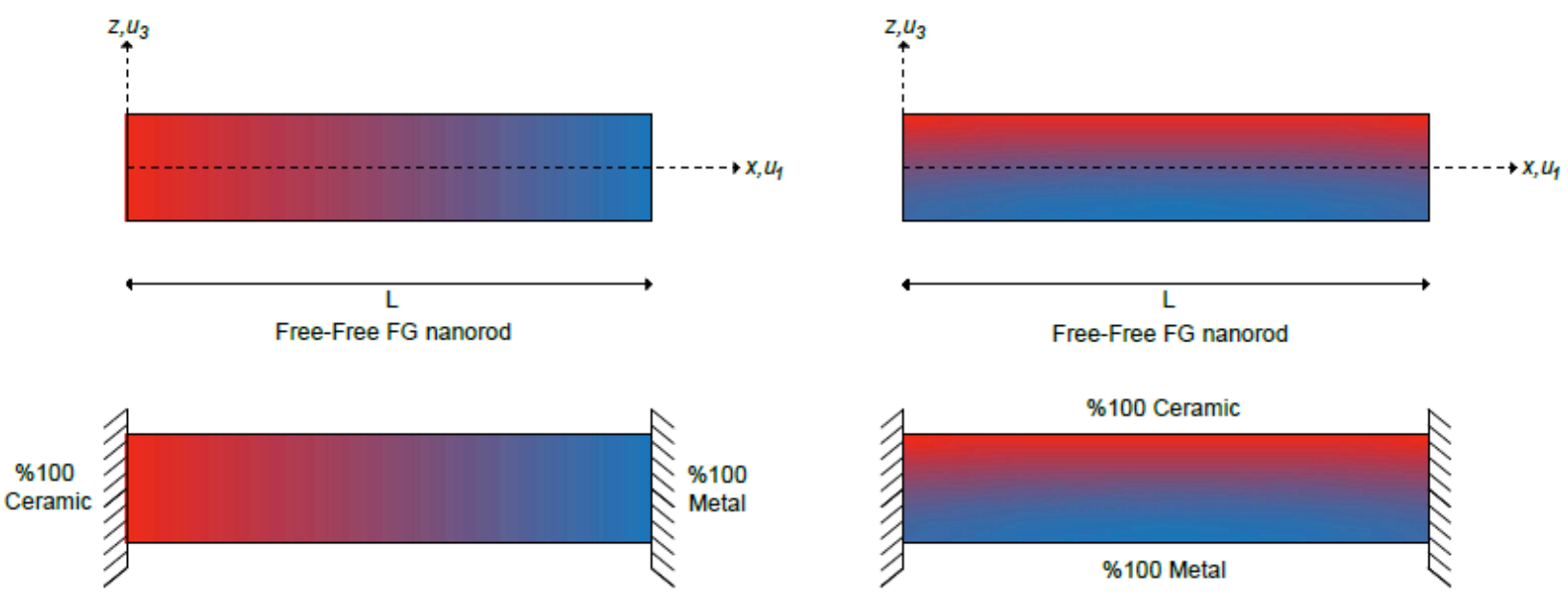

Clamped-Clamped FG nanorod

Clamped-Clamped FG nanorod
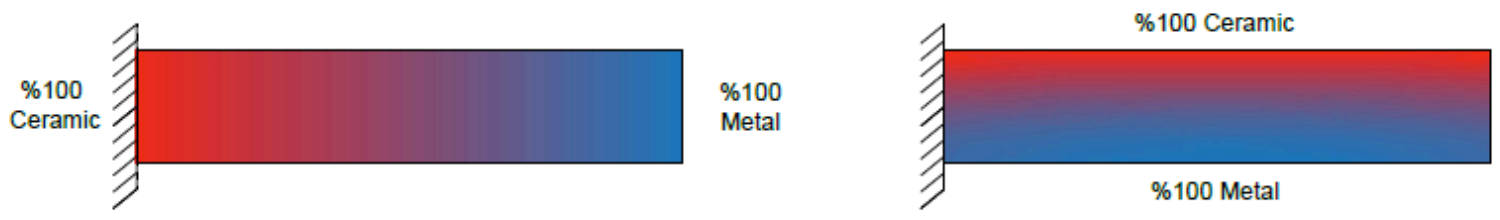

Clamped-Free FG nanorod

Clamped-Free FG nanorod

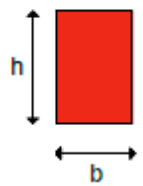

Section I

a) Type - I

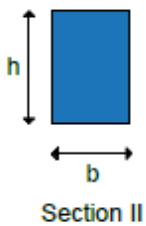

Section II

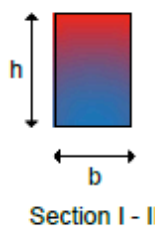

b) Type - II

Fig. 1. Functionally graded nanorods with various boundary conditions 


\section{Functionally Graded Rod}

FG nanorods with various boundary conditions like free-free, clamped-clamped and clampedfree are illustrated in Figure 1. $L, b$ and $h$ represent the length, width and thickness of the FG rod, respectively. Type I (Fig. 1a) and Type II (Fig. 1b) represent the FG nanorods whose material properties vary continuously in the axial direction and thickness direction, respectively. The material properties such as Young's modulus, density etc. change of the rod according to a power-law. If the changing of material properties of the rod is assumed in the thickness direction, the effective material properties of rod can be defined as $[11,13]$

$$
P(z)=\left(P_{c}-P_{m}\right)\left(\frac{z}{h}+\frac{1}{2}\right)^{k}+P_{m}
$$

Where, $P$ represents the effective material property, while $k$ represents the non-negative power-law exponent. The subscripts $c$ and $m$ indicate the ceramic and metal materials, respectively. $u_{1}, u_{2}$ and $u_{3}$ are the displacements of the FG rod in the $x, y, z$ directions, respectively, and may be written as follow

$$
u_{1}(x, z, t)=u(x, t), \quad u_{2}(x, z, t)=0, u_{3}(x, z, t)=0
$$

$u$, and $t$ denote the axial displacement of any point on the neutral axis and time, respectively. Stress $(\sigma)$ and normal force $(N)$ expressions for the FG rod are written as follows

$$
\begin{aligned}
& \sigma_{x x}=E(z) \varepsilon_{x x} \\
& N=\int_{A} \sigma_{x x}(z) d A
\end{aligned}
$$

Here, $\varepsilon$ and $A$ are strain and cross-section area, respectively. The equations of motions of FG nano-sized rod can be obtained by means of the Hamilton's principle [36]

$$
\int_{t_{1}}^{t_{2}}(\delta K-\delta U+\delta W) d t=0
$$

Where $U, K$ and $W$ are the strain energy, kinetic energy and work done by external forces, respectively. The external loads can be encountered as elastic foundation, axial compressive force, thermal loading etc. However, there are no external forces in this vibration problem of FG nanorod and so $W$ is set to zero. The first variations of the strain energy and kinetic energy are given as follows 


$$
\begin{gathered}
\delta \int_{t_{1}}^{t_{2}} U d t=\int_{t_{1}}^{t_{2} L} N \delta\left(\frac{\partial u}{\partial x}\right) d x d t \\
\delta \int_{t_{1}}^{t_{2}} K d t=\int_{t_{1}}^{t_{2} L} \int_{0} I_{0} \frac{\partial u}{\partial t} \delta\left(\frac{\partial u}{\partial t}\right) d x d t
\end{gathered}
$$

Here, $I_{0}$ is expressed as

$$
I_{0}=\int_{A} \rho(z) d A
$$

By substituting equations (6) - (7) into equation (5) and after some mathematical arrangements, we obtain the equation of motion of the rod as follows

$$
\delta u: \frac{\partial N}{\partial x}=I_{0} \frac{\partial^{2} u}{\partial t^{2}}
$$

\section{Size-Dependent Finite Element Formulation}

The nonlocal constitutive formulation is [37]

$$
\left[1-\left(e_{0} a\right)^{2} \nabla^{2}\right] \sigma_{i j}=C_{i j k l} \varepsilon_{k l}
$$

Where $\sigma_{i j}$ is the stress tensor, $C_{i j k l}$ is the fourth-order Young's modulus tensor, $\varepsilon_{k l}$ is the strain tensor, $e_{0} a$ is the nonlocal parameter. The Equation (10) can be rewritten as

$$
\sigma_{x x}-\left(e_{0} a\right)^{2} \frac{\partial^{2} \sigma_{x x}}{\partial x^{2}}=E(z) \varepsilon_{x x}
$$

Integrating Eq. (11) over the cross-section area, we obtain the axial force-strain relation as Eq. (12)

$$
N-\left(e_{0} a\right)^{2} \frac{\partial^{2} N}{\partial x^{2}}=A_{1} \frac{\partial u}{\partial x}
$$

Here, $A_{l}$ is expressed as

$$
A_{1}=\int_{A} E(z) d A
$$

Differentiating Equation (9) with respect to $x$, then substituting into Equation (12) we obtain Equation (14).

$$
N=A_{1} \frac{\partial u}{\partial x}+\left(e_{0} a\right)^{2} I_{0} \frac{\partial^{3} u}{\partial x \partial t^{2}}
$$


By substituting Equation (14) into Equation (9), the equation of the motion of FG nanorod is obtained as

$$
A_{1} \frac{\partial^{2} u}{\partial x^{2}}+\left(e_{0} a\right)^{2} I_{0} \frac{\partial^{4} u}{\partial x^{2} \partial t^{2}}-I_{0} \frac{\partial^{2} u}{\partial t^{2}}=0
$$

In this study, a rod finite element is considered has two nodes. $\phi$ is the interpolation (or shape) functions matrix of a rod finite element and expressed as below

$$
[\phi]=\left[\begin{array}{ll}
1-\frac{x}{L} & \frac{x}{L}
\end{array}\right]
$$

The stiffness matrix, classical mass and nonlocal mass matrices are obtained using Eqs. (15) (16) as follows

$$
\begin{gathered}
K=\int_{0}^{L} A_{1}\left([\phi]^{\prime}\right)^{T}[\phi]^{\prime} d x \\
M_{c l}=\int_{0}^{L} I_{0}([\phi])^{T}[\phi] d x \\
M_{n l}=\left(e_{0} a\right)^{2} \int_{0}^{L} I_{0}\left([\phi]^{\prime}\right)^{T}[\phi]^{\prime} d x
\end{gathered}
$$

In the above Equations, superscript $T$ represents the transpose operator. The subscripts $c l$ and $n l$ are used to indicate the classical and nonlocal theories, respectively. The frequencies of FG nano-sized rod are found as follows

$$
\left|K-\omega_{n}^{2}\left(M_{n l}+M_{c l}\right)\right|=0
$$

Here $\omega_{n}$ and the subscript $n$ indicate the circular frequency and mode number.

\section{Numerical Results}

In this section, comparison studies and numerical examples are performed. Comparison studies are presented by $\mathrm{Xu}$ et al. [38] and Numanoğlu et al. [39]. Table 1 is presented to compare the validity of the method and to show the compatibility with each other. Comparisons of non-dimensional frequencies for the first four modes of clamped-free homogeneous nanorods are shown in Table 1. Also, this Table demonstrates the effect of the number of finite element $(N)$ on convergence. As can be seen, the number of finite elements is an important issue for the convergence of frequency values. The appropriate number of elements should be chosen to ensure desired convergence. As can be seen, low number of finite elements provides the desired convergence for low modes. However, it may be 
necessary to increase the number of finite elements as the mode number increase. Dimensionless parameters used in the comparison studies are defined as follows

$$
\bar{\omega}_{n}=\omega_{n} L \sqrt{\rho / E}, \quad \bar{\mu}=e_{0} a / L
$$

Table 1. Comparison of dimensionless frequencies of homogeneous nanorod

\begin{tabular}{|c|c|c|c|c|c|c|c|}
\hline $\bar{\mu}$ & $\bar{\omega}_{n}$ & Xu et al. [38] & $\begin{array}{l}\text { Numanoğlu et } \\
\text { al. } \\
{[39]}\end{array}$ & $\begin{array}{l}\text { Present } \\
\text { study } \\
\quad(\mathrm{N}=200) \\
\end{array}$ & $\begin{array}{l}\text { Present study } \\
\quad(\mathrm{N}=100)\end{array}$ & $\begin{array}{l}\text { Present } \\
\text { study } \\
\quad(\mathrm{N}=50) \\
\end{array}$ & $\begin{array}{l}\text { Present study } \\
\quad(\mathrm{N}=20)\end{array}$ \\
\hline \multirow[t]{4}{*}{0.0} & $n=1$ & 1.57080 & 1.57080 & 1.5708 & 1.5708 & 1.5709 & 1.5712 \\
\hline & $n=2$ & 4.71239 & 4.71239 & 4.7125 & 4.7128 & 4.7141 & 4.7233 \\
\hline & $n=3$ & 7.85398 & 7.85398 & 7.8545 & 7.8560 & 7.8621 & 7.9045 \\
\hline & $n=4$ & 10.99557 & 10.99557 & 10.9970 & 11.0011 & 11.0177 & 11.1345 \\
\hline \multirow{4}{*}{0.1} & $n=1$ & 1.55177 & 1.55177 & 1.5518 & 1.5518 & 1.5518 & 1.5522 \\
\hline & $n=2$ & 4.26279 & 4.26279 & 4.2629 & 4.2631 & 4.2641 & 4.2709 \\
\hline & $n=3$ & 6.17668 & 6.17668 & 6.1769 & 6.1777 & 6.1806 & 6.2012 \\
\hline & $n=4$ & 7.39805 & 7.39805 & 7.3985 & 7.3997 & 7.4048 & 7.4399 \\
\hline \multirow{4}{*}{0.2} & $n=1$ & 1.49858 & 1.49858 & 1.4986 & 1.4986 & 1.4986 & 1.4989 \\
\hline & $n=2$ & 3.42933 & 3.42933 & 3.4294 & 3.4295 & 3.4300 & 3.4335 \\
\hline & $n=3$ & 4.21782 & 4.21782 & 4.2179 & 4.2181 & 4.2191 & 4.2256 \\
\hline & $n=4$ & 4.55152 & 4.55152 & 4.5516 & 4.5519 & 4.5531 & 4.5612 \\
\hline
\end{tabular}

In this section, effects of power-law exponent and the nonlocal parameter on the free vibration response of functionally graded nanorod are investigated. In the numerical calculations, the number of finite elements for FG nanorod is chosen as 200. Functionally graded nanorod is considered composed of aluminum and alumina and with clamped-free boundary condition. The top and bottom surfaces of the nanorod are composed of pure alumina (ceramic) and aluminum (metal), respectively. Mechanical properties of functionally graded nanorod constituents are given as [40]: $E_{\mathrm{m}}=70 \mathrm{GPa}, \rho_{\mathrm{m}}=2700 \mathrm{~kg} / \mathrm{m}^{3}$ for aluminum and $\mathrm{E}_{\mathrm{c}}=393 \mathrm{Gpa}$, $\rho_{\mathrm{c}}=3960 \mathrm{~kg} / \mathrm{m}^{3}$ for alumina. The following dimensionless frequency parameter is used

$$
\lambda_{n}=\omega_{n} L \sqrt{\rho_{c} / E_{c}}
$$




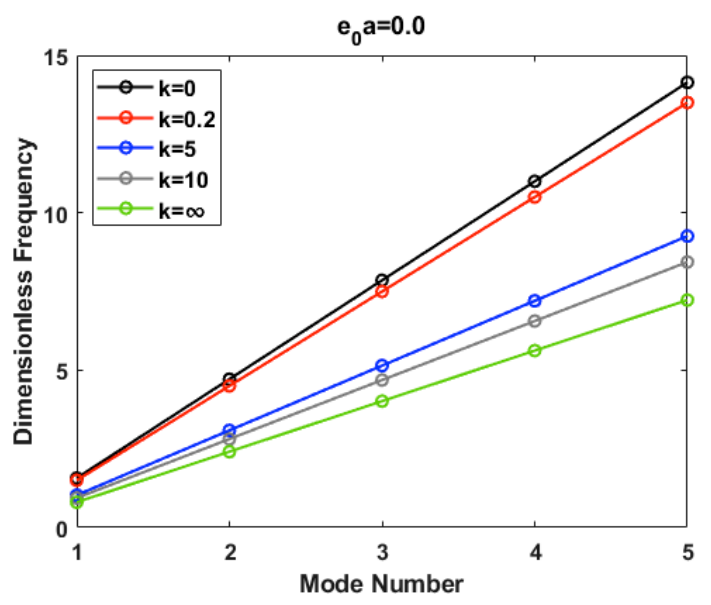

(a)

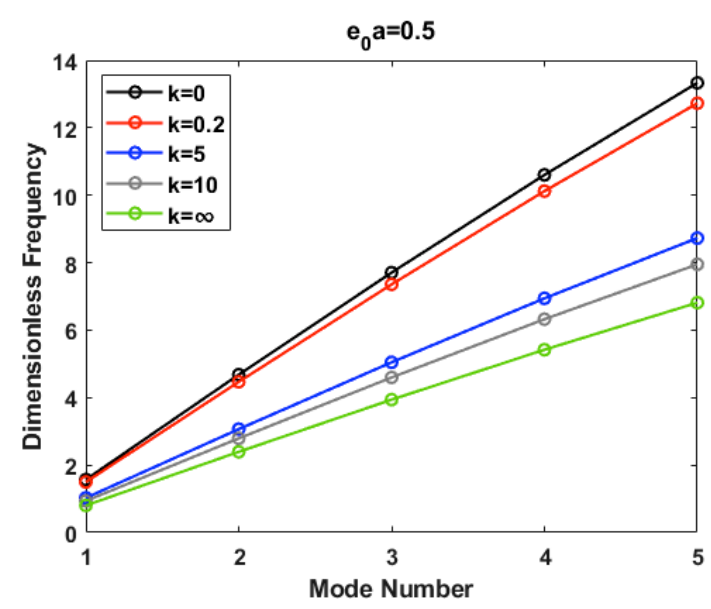

(b)

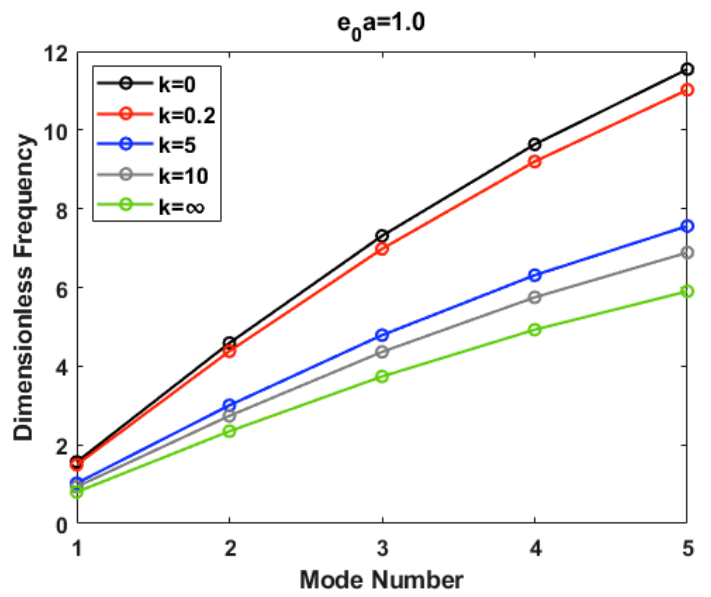

(c)

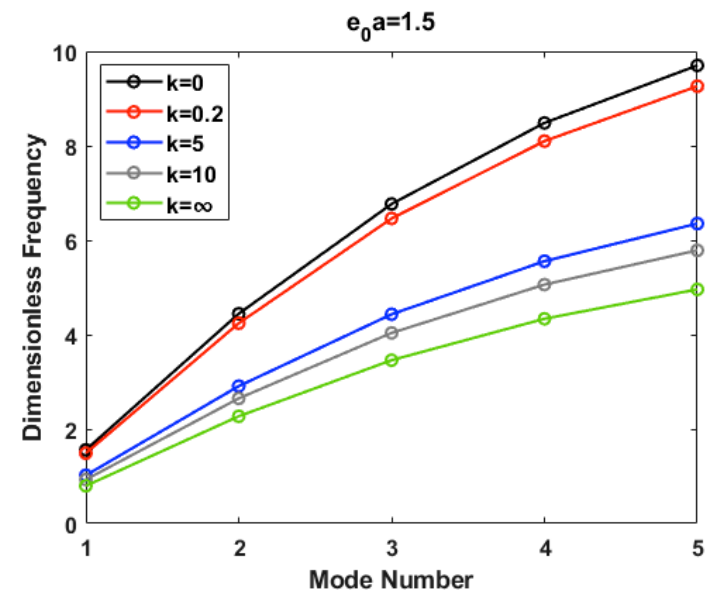

(d)

Fig. 2. Variation of dimensionless frequencies of FG nanorod

Figure 2 displays the variation of dimensionless frequencies of functionally graded nanorod with respect to mode numbers for various power-law exponent $(k)$ and nonlocal parameter $\left(e_{0} a\right)$ values. The Figure 2 is plotted from the analyses of FG nanorod with various nonlocal parameters ranging from 0 to 1.5 and various power-law exponents ranging from 0 to $\infty$. It is concluded from the Figure that the increasing values of power-law exponent and nonlocal parameter lead to a decrease in the dimensionless frequencies of FG nanorods. It should be noted that when the power-law exponent set to zero $(\mathrm{k}=0)$, the results give the frequencies of alumina (pure ceramic). If the power-law exponent sets to infinity $(\mathrm{k}=\infty)$, the frequencies of aluminum (pure metal) are obtained. Also, if the nonlocal parameter $\mathrm{e}_{0} \mathrm{a}$ set to zero, the frequencies of the classical theory are obtained. 


\section{Conclusions}

In the present study, the nonlocal finite element formulation of functionally graded nanorod is proposed in conjunction with Eringen's nonlocal elasticity theory. The stiffness and mass matrices essential to the vibration response of functionally graded nanorod are found using interpolation functions. Finally, an eigenvalue problem is defined with the obtained matrices and $\omega_{n}$, and the eigenvalues $\omega_{n}$ are found by setting the determinant of the coefficient matrix to zero. A numerical example for clamped-free boundary condition is given to investigate the influences of some parameters on frequencies of FG nanorod. The main results obtained in this study can be summarized as follows: When the nonlocal effect is ignored, that is when the $\mathrm{e}_{0} \mathrm{a}$ value is taken as zero, the frequencies of the FG nanorod have the highest values. It is understood from that the nonlocal effect causes a reduction in the frequency of the FG nanorod. In addition, it is seen that with the increase of the power-law exponent value, that is with the transition of material properties from ceramic to metal, there is a decrease in frequencies.

\section{References}

[1] Lü, C.F., Lim, C.W. and Chen, W.Q., Size-dependent elastic behavior of FGM ultra-thin films based on generalized refined theory. International Journal of Solids and Structures, 46(5), 1176-1185, 2009.

[2] Lanhe, W., Thermal buckling of a simply supported moderately thick rectangular FGM plate. Composite Structures, 64(2), 211-218, 2004.

[3] Belkorissat, I., Houari, M.S.A., Tounsi, A., Bedia, E.A. and Mahmoud, S.R., On vibration properties of functionally graded nano-plate using a new nonlocal refined four variable model. Steel and Composite Structures, 18(4), 1063-1081, 2015.

[4] Żur, K.K., Arefi, M., Kim, J. and Reddy, J.N., Free vibration and buckling analyses of magneto-electro-elastic FGM nanoplates based on nonlocal modified higher-order sinusoidal shear deformation theory. Composites Part B: Engineering, 182, 107601, 2020.

[5] Ebrahimi, F., Ehyaei, J. and Babaei, R. Thermal buckling of FGM nanoplates subjected to linear and nonlinear varying loads on Pasternak foundation. Advances in materials Research, 5(4), 245, 2016.

[6] Yuan, Y., Zhao, K., Sahmani, S. and Safaei, B. Size-dependent shear buckling response of FGM skew nanoplates modeled via different homogenization schemes. Applied Mathematics and Mechanics, 1-18, 2020.

[7] Karami, B., Shahsavari, D., Janghorban, M. and Li, L. On the resonance of functionally graded nanoplates using bi-Helmholtz nonlocal strain gradient theory. International Journal of Engineering Science, 144, 103143, 2019.

[8] Uzun, B. and Yayl1, M.Ö. Nonlocal vibration analysis of Ti-6Al-4V/ZrO functionally graded nanobeam on elastic matrix. Arabian Journal of Geosciences, 13(4), 1-10, 2020.

[9] Uzun, B., Yayl1, M.Ö. and Deliktaş, B. Free vibration of FG nanobeam using a finiteelement method. Micro \& Nano Letters, 15(1), 35-40, 2020.

[10] Uzun, B. and Yayl1, M.Ö., Finite element model of functionally graded nanobeam for free vibration analysis. International Journal of Engineering and Applied Sciences, 11(2), 387-400, 2019. 
[11] Hosseini, S.A.H. and Rahmani, O., Free vibration of shallow and deep curved FG nanobeam via nonlocal Timoshenko curved beam model. Applied Physics A, 122(3), 169, 2016.

[12] Jalaei, M.H. and Civalek, Ö., On dynamic instability of magnetically embedded viscoelastic porous FG nanobeam. International Journal of Engineering Science, 143, 1432, 2019.

[13] Saffari, S., Hashemian, M. and Toghraie, D., Dynamic stability of functionally graded nanobeam based on nonlocal Timoshenko theory considering surface effects. Physica B: Condensed Matter, 520, 97-105, 2017.

[14] Aydogdu, M., Arda, M. and Filiz, S., Vibration of axially functionally graded nano rods and beams with a variable nonlocal parameter. Advances in nano research, 6(3), 257, 2018.

[15] Arda, M., Axial dynamics of functionally graded Rayleigh-Bishop nanorods. Microsystem Technologies, 1-14, 2020.

[16] Kiani, K., Free dynamic analysis of functionally graded tapered nanorods via a newly developed nonlocal surface energy-based integro-differential model. Composite Structures, 139, 151-166, 2016.

[17] Arefi, M. and Zenkour, A.M., Employing the coupled stress components and surface elasticity for nonlocal solution of wave propagation of a functionally graded piezoelectric Love nanorod model. Journal of Intelligent Material Systems and Structures, 28(17), 2403-2413, 2017.

[18] Narendar, S., Wave dispersion in functionally graded magneto-electro-elastic nonlocal rod. Aerospace Science and Technology, 51, 42-51 2016.

[19] Akgöz, B. and Civalek, Ö., A size-dependent beam model for stability of axially loaded carbon nanotubes surrounded by Pasternak elastic foundation. Composite Structures, 176, 1028-1038, 2017.

[20] Trinh, L.C., Vo, T.P., Thai, H.T. and Nguyen, T.K. An analytical method for the vibration and buckling of functionally graded beams under mechanical and thermal loads. Composites Part B: Engineering, 100, 152-163, 2016.

[21] Civalek, Ö. and Kiracioglu, O. (2010). Free vibration analysis of Timoshenko beams by DSC method. International Journal for Numerical Methods in Biomedical Engineering, 26(12), 1890-1898, 2010.

[22] Civalek, O. and Yavas, A., Large deflection static analysis of rectangular plates on two parameter elastic foundations. International journal of science and technology, 1(1), 4350, 2006.

[23] Civalek, Ö., Geometrically non-linear static and dynamic analysis of plates and shells resting on elastic foundation by the method of polynomial differential quadrature (PDQ), Ph. D. Thesis, Firat University, Elazig, 2004 (in Turkish), 2004.

[24] Civalek, Ö., Finite Element analysis of plates and shells, Elazı̆̆: Firat University,1998.

[25] Demir, Ç. and Civalek, Ö., A new nonlocal FEM via Hermitian cubic shape functions for thermal vibration of nano beams surrounded by an elastic matrix. Composite Structures, 168, 872-884, 2017.

[26] Adhikari, S., Murmu, T. and McCarthy, M.A., Dynamic finite element analysis of axially vibrating nonlocal rods. Finite Elements in Analysis and Design, 63, 42-50, 2013.

[27] Hemmatnezhad, M. and Ansari, R., Finite element formulation for the free vibration analysis of embedded double-walled carbon nanotubes based on nonlocal Timoshenko beam theory. Journal of theoretical and applied physics, 7(1), 6, 2013.

[28] Civalek, Ö., Uzun, B., Yayl, M.Ö. and Akgöz, B. Size-dependent transverse and longitudinal vibrations of embedded carbon and silica carbide nanotubes by nonlocal finite element method. The European Physical Journal Plus, 135(4), 381, 2020. 
[29] Ghannadpour, S.A.M., A variational formulation to find finite element bending, buckling and vibration equations of nonlocal Timoshenko beams. Iranian Journal of Science and Technology, Transactions of Mechanical Engineering, 43(1), 493-502, 2019.

[30] Akbaş, Ş.D., Static, Vibration, and Buckling Analysis of Nanobeams. Nanomechanics, $123,2017$.

[31] Anjomshoa, A., Shahidi, A.R., Hassani, B. and Jomehzadeh, E. Finite element buckling analysis of multi-layered graphene sheets on elastic substrate based on nonlocal elasticity theory. Applied Mathematical Modelling, 38(24), 5934-5955, 2014.

[32] Taghizadeh, M., Ovesy, H.R. and Ghannadpour, S.A.M. Beam buckling analysis by nonlocal integral elasticity finite element method. International Journal of Structural Stability and Dynamics, 16(06), 1550015, 2016.

[33] Demir, C., Mercan, K., Numanoglu, H.M. and Civalek, O., Bending response of nanobeams resting on elastic foundation. Journal of Applied and Computational Mechanics, 4(2), 105-114, 2018.

[34] Taghizadeh, M., Ovesy, H.R. and Ghannadpour, S.A.M., Nonlocal integral elasticity analysis of beam bending by using finite element method. Structural Engineering and Mechanics, 54(4), 755-769, 2015.

[35] Mahmoud, F.F., Eltaher, M.A., Alshorbagy, A.E. and Meletis, E.I., Static analysis of nanobeams including surface effects by nonlocal finite element. Journal of mechanical science and technology, 26(11), 3555-3563, 2012.

[36] Reddy, J.N., Energy Principles and Variational Methods in Applied Mechanics, John Wiley \& Sons, 2nd Edition, 2002.

[37] Eringen, A.C., On differential equations of nonlocal elasticity and solutions of screw dislocation and surface waves. Journal of applied physics, 54(9), 4703-4710, 1983.

[38] Xu, X.J., Zheng, M.L. and Wang, X.C. On vibrations of nonlocal rods: Boundary conditions, exact solutions and their asymptotics. International Journal of Engineering Science, 119, 217-231, 2017.

[39] Numanoğlu, H.M., Akgöz, B. and Civalek, Ö. On dynamic analysis of nanorods. International Journal of Engineering Science, 130, 33-50, 2018.

[40] Asghari, M., Rahaeifard, M., Kahrobaiyan, M.H. and Ahmadian, M.T., The modified couple stress functionally graded Timoshenko beam formulation. Materials \& Design, 32(3), 1435-1443, 2011. 